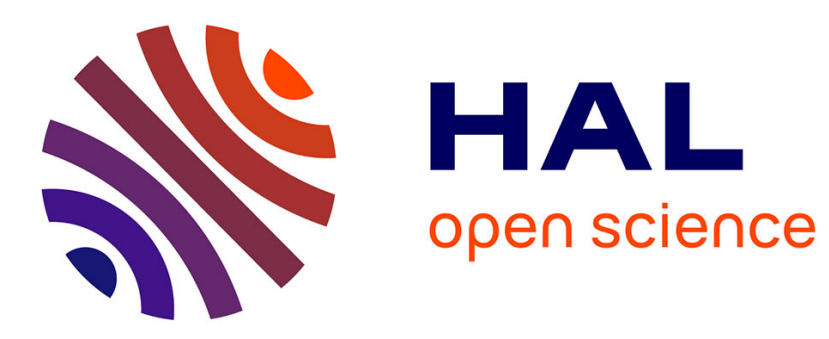

\title{
Physical modelling of trombone mutes, the pedal note issue
}

\author{
Lionel Velut, Christophe Vergez, Joël Gilbert
}

\section{To cite this version:}

Lionel Velut, Christophe Vergez, Joël Gilbert. Physical modelling of trombone mutes, the pedal note issue. Acta Acustica united with Acustica, 2017, 103 (4), pp.668-675. hal-01385524v2

\section{HAL Id: hal-01385524 \\ https://hal.science/hal-01385524v2}

Submitted on 22 Feb 2017

HAL is a multi-disciplinary open access archive for the deposit and dissemination of scientific research documents, whether they are published or not. The documents may come from teaching and research institutions in France or abroad, or from public or private research centers.
L'archive ouverte pluridisciplinaire HAL, est destinée au dépôt et à la diffusion de documents scientifiques de niveau recherche, publiés ou non, émanant des établissements d'enseignement et de recherche français ou étrangers, des laboratoires publics ou privés. 


\title{
- Physical modelling of trombone mutes, the pedal note issue
}

\section{${ }_{27}$ I Introduction}

\author{
Lionel Velut ${ }^{1}$, Christophe Vergez ${ }^{1}$, and Joël Gilbert ${ }^{2}$
}

${ }^{1}$ LMA, CNRS, UPR 7051, Aix-Marseille Univ., Centrale Marseille, F-13453

Marseille cedex 13, France.

${ }^{2}$ Laboratoire d'Acoustique de l'Université du Maine, UMR CNRS-6613, Avenue Olivier Messiaen, 72085 Le Mans cedex 9, France

\begin{abstract}
Brass players use a variety of mutes to change the sound of their instrument for artistic expression. However, mutes can also modify the intonation and the playability of the muted instrument. An example is the use of a straight mute on a trombone, which makes it very difficult to play stable pedal notes.

Previous studies have shown that using a straight mute establishes a subsidiary acoustic resonance in the trombone. To cancel this modification, an active control device was developed and integrated into a mute, with satisfying experimental results [Meurisse et al., 2015]. With this device, the perturbed pedal notes can easily be played again.

This paper investigates the ability of a physical model of brass instrument to reproduce the behaviour of the trombone pedal $B b$ without mute, or with an "active" or a "passive" straight mute. Linear stability analysis and time-domain simulations are used to analyse the behaviour of the model in the parameter range corresponding to the pedal note. Numerical results are compared for different models of instruments: a trombone, a trombone with a straight (passive) mute, an a trombone with an active mute. It is shown that the simple physical model considered behaves rather qualitatively similarly to what is experienced with real instruments: the playing of the pedal note is perturbed with a passive mute, whereas the model of trombone with the experimental active mute gives results very similar to those obtained with an open trombone.
\end{abstract}

A usual solution for changing the timbre of a brass instrument consists in using a mute, which is a device plugged to the opening of the instrument bell, or held by hand just in front of the bell. The 
shape and the material of the mute affect the pressure radiation of the bell, therefore modifying the emitted sound [Campbell and Greated, 1994, p.398]. As a side effect, introducing an obstacle in the bell, or close to it, also modifies the acoustical properties of the instrument [Backus, 1976]. This has various consequences, including modification of the instrument tuning and of reported instrument-player interaction.

Pedal notes are the lowest playable notes on a trombone. When the slide is fully closed, the note played is a $B b 1$, corresponding to a playing frequency of $58 \mathrm{~Hz}$ in equal temperament. In brass instruments, most regimes of oscillation of the instrument have a playing frequency slightly above the acoustical resonance supporting the oscillation. However, the pedal note has a playing frequency unusually far above the resonance frequency of the acoustical mode supporting the oscillation, making it a particular regime of oscillation as detailed in [Gilbert and Aumond, 2008, Velut et al., 2017]. Furthermore, the pedal note oscillation is supported by the lowest mode of the trombone, which is inharmonic with the other modes of the trombone as shown in Fig. 2. When a straight mute is inserted in the trombone, playing stable pedal notes on the three first slides positions - $B b 1, A^{1}$ and $A b^{1}$ - is uneasy and results in a rolling, unstable sound [Sluchin and Caussé, 1991, Meurisse et al., 2015]. Measurements of the input impedance of a trombone with a mute [Meurisse et al., 2015, Velut et al., 2016b] show the occurrence of a subsidiary acoustical mode between the first and the second modes.

This paper will particularly focus on the pedal $B b 1$, corresponding to the slide in the shortest position. It will hereinafter be referred to as "the pedal note". An active control device has been previously developed to remove this subsidiary mode [Meurisse et al., 2015], which makes it possible to play the pedal note with a straight mute. It consists of an "active mute", a commercial straight mute equipped with an active control device which cancels the aforesaid subsidiary resonance mode.

The purpose of this paper is to investigate to what extent a simple trombone physical model can predict the effect of a trombone straight mute on the pedal note and the effectiveness of the active mute. The physical model of a brass instrument is first presented. Then, linear stability analysis (LSA) and time-domain simulations are used to analyse the behaviour of the pedal note in this model. Analyses are conducted on an "open trombone" configuration (tenor trombone without any mute), a "passive mute" configuration (the same trombone with a commercial straight mute) and an "active mute" configuration (the same trombone with an identical straight mute and the described active control loop). Results of this model are compared with the experimental results from [Meurisse et al., 2015]. 


\section{Tools}

\section{II.A Brass instrument model}

A physical model of trombone, suitable for a large class of music instruments, is presented in this article. Following Helmholtz pioneering work [von Helmholtz, 1870], the trombone is modelled as a closed-loop system consisting of an exciter and a resonator which are coupled, as illustrated in Fig. 1. Such a system can produce auto-oscillation on different regimes.

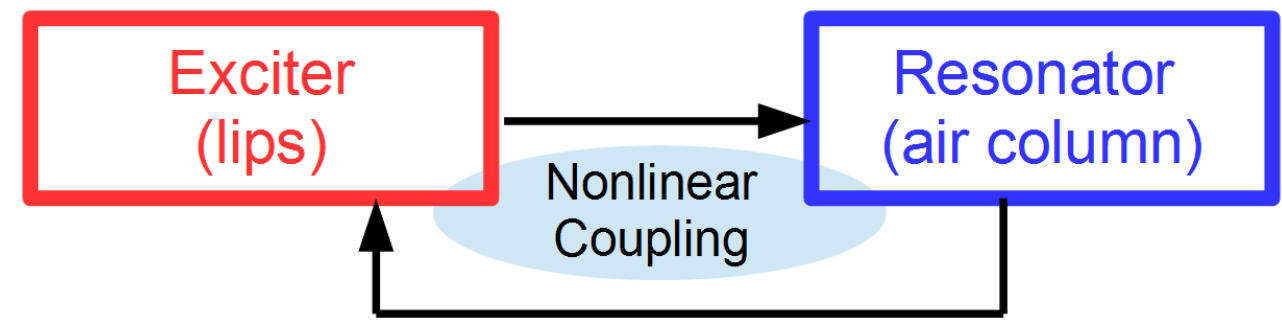

Figure 1: Closed-loop model in free oscillation, suitable for the description of most self-sustained musical instruments, including trombones. Self-sustained oscillations are generated by the localised non-linear coupling (here the airflow between lips) between a linear exciter (here the lips) and a linear resonator (here the air column inside the instrument bore).

For a brass instrument, the exciter is the lips of the musician, which act as a valve: the section of the channel between the lips depends on the pressure difference through these lips as well as on their mechanical characteristics. Multiple models of the lip reed have been proposed and used, with one degree of freedom [Eliott and Bowsher, 1982, Fletcher, 1993, Cullen et al., 2000, Silva et al., 2007] or 2 DOF [Adachi and Sato, 1996, Campbell, 2004, Lopez et al., 2006, Newton et al., 2008]. The model used for this paper is the one-DOF valve model, usually referred to as the "outward-striking" model, also called $(+,-)$ swinging-door model in the literature:

$$
\frac{d^{2} h}{d t^{2}}+\frac{\omega_{l}}{Q_{l}} \frac{d h}{d t}+\omega_{l}^{2}\left(h-h_{0}\right)=\frac{1}{\mu}\left(p_{b}-p(t)\right),
$$

where $h$ is the height of the lip channel $(\mathrm{m}) ; p$ is the pressure at the input of the instrument, in the mouthpiece $(\mathrm{Pa}) ; p_{b}$ is the constant blowing pressure in the mouth $(\mathrm{Pa}) ; \omega_{l}=2 \pi f_{l}\left(\mathrm{rad} \cdot \mathrm{s}^{-1}\right)$ is the lip resonance angular frequency; $Q_{l}$ is the (dimensionless) quality factor of the lips; $h_{0}$ is the value of $h(t)$ at rest; $\mu$ is an equivalent surface mass $\left(\mathrm{kg} \cdot \mathrm{m}^{-2}\right)$.

Although it does not fully reproduce all the observed behaviours of human or artificial lips, this model is sufficient for reproducing the normal playing situations [Yoshikawa, 1995], including the pedal note of the trombone [Velut et al., 2017] and multiphonic sounds [Velut et al., 2016a]. As a limitation, this model is known to oscillate at higher frequencies than those at which a musician would play on the same acoustical mode. Even for this relatively simple model, choosing the lip parameters is challenging and requires a thorough bibliographical review. This was conducted in [Velut et al., 2017]. The resulting set of parameters is given in table I. 


\begin{tabular}{|c|c|c|c|}
\hline$h_{0}(\mathrm{~m})$ & $W(\mathrm{~m})$ & $1 / \mu\left(\mathrm{m}^{2} \cdot \mathrm{kg}^{-1}\right)$ & $Q_{l}$ \\
\hline $5 \times 10^{-4}$ & $12 \times 10^{-3}$ & 0.11 & 7 \\
\hline
\end{tabular}

The resonator is the air column contained in the bore of the instrument. Given the low playing amplitude considered in this article, the brassiness phenomenon, related to non-linear propagation in the instrument [Myers et al., 2012] is not taken into account. Under this hypothesis, the resonator can be fully described by its input impedance, which is by definition the ratio of the pressure $P(\omega)$ to the flow $U(\omega)$ at the input of the instrument, in the frequency domain:

$$
Z(\omega)=\frac{P(\omega)}{U(\omega)} .
$$

This value can be measured using the sensor described in [Macaluso and Dalmont, 2011]. In this paper, three input impedance measurements are used: the impedance of an open trombone (without any mute), the impedance of the same trombone with a "passive mute" (mute without active control) and the impedance of this trombone with an "active mute", with the feedback active control device enabled.

The input impedance can be considered as a sum of peaks, each peak corresponding to a resonance mode of the air column inside the instrument. Thus, it can be fitted with a sum of complex modes, corresponding to a sum of poles-residues functions:

$$
Z(\omega)=Z_{c} \cdot \sum_{n=1}^{N_{m}}\left[\frac{C_{n}}{j \omega-s_{n}}+\frac{C_{n}^{*}}{j \omega-s_{n}^{*}}\right],
$$

$C_{n}$ and $s_{n}$ being the dimensionless complex residues and poles of the complex modes of the fitted impedance, respectively. $Z_{c}=\frac{\rho . c}{\pi \cdot r^{2}}$ is the characteristic impedance of the resonator, $\rho$ is the air density, $c$ the celerity of acoustic waves in the air and $r$ the input radius of the mouthpiece. $N_{m}$ is the number of modes used to fit the impedance, fixed to $N_{m}=13$ in this article. Translation of eq. (3) in the time domain leads to an ordinary differential equation for each complex modal component $p_{n}$ of the pressure $p(t)$ :

$$
\frac{d p_{n}}{d t}=s_{n} p_{n}(t)+Z_{c} C_{n} \cdot u(t) \quad \forall n \in\left[1 . . N_{m}\right],
$$

where $u(t)$ is the time-domain expression of the flow at the input of the instrument. Furthermore, $p(t)=2 \sum_{n=1}^{N_{m}} \Re\left[p_{n}(t)\right]$. Details of this modal formulation of the pressure, already used in [Velut et al., 2017], can be found in [Silva, 2009].

The fit is optimised by a least mean squares algorithm. This results in a very good match between the measured impedance and the fit, as shown in Fig. 2. 

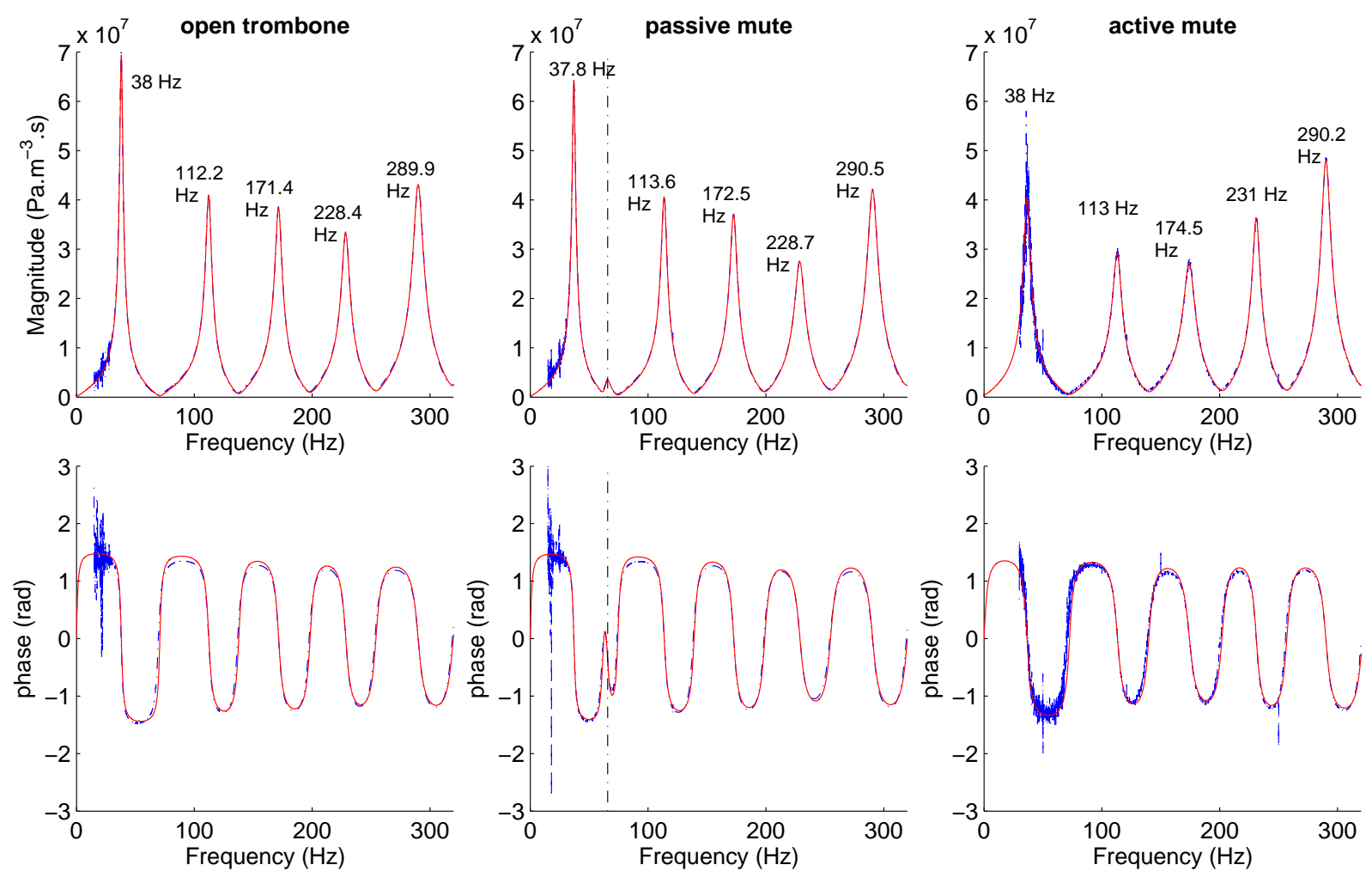

Figure 2: (colour online) Comparison of the measured impedances (blue, dash-dotted) and their modal fits (red, plain) with 13 complex modes. Magnitudes (top plots) and phases (bottom plots) of the impedances for the three situations - open trombone (left), passive straight mute (middle) and active mute (right) - are displayed. The dash-dotted line at $65.7 \mathrm{~Hz}$ indicates the subsidiary resonance. The resonance frequencies of the other modes are written near the amplitude peaks.

The lips and the resonator are coupled through the expression of the flow $u(t)$ of the air jet through the lip channel:

$$
u(t)=W \cdot h(t) \cdot \sqrt{\frac{2 \cdot\left|p_{b}-p(t)\right|}{\rho}} \cdot \operatorname{sign}\left(p_{b}-p(t)\right) \cdot \theta(h),
$$

where $W$ is the width of the lip channel and $\rho$ the air density, sign is the sign function and $\theta(h)$ is the Heaviside step function. This non-linear expression of the flow was proposed in [Wilson and Beavers, 1974, Eliott and Bowsher, 1982] and has been used in almost every publication about brasswind and woodwind physical models since.

The whole model can therefore be written:

$$
\left\{\begin{aligned}
\frac{d^{2} h}{d t^{2}}+\frac{\omega_{l}}{Q_{l}} \frac{d h}{d t}+\omega_{l}^{2}\left(h-h_{0}\right) & =\frac{1}{\mu}\left(p_{b}-p(t)\right) \\
u(t) & =W \cdot h(t) \cdot \sqrt{\frac{2 \cdot\left|p_{b}-p(t)\right|}{\rho}} \cdot \operatorname{sign}\left(p_{b}-p(t)\right) \cdot \theta(h) \\
\frac{d p_{n}}{d t} & =s_{n} p_{n}(t)+Z_{c} C_{n} \cdot u(t) \quad \forall n \in\left[1 . . N_{m}\right] \\
p(t) & =2 \sum_{n=1}^{N_{m}} \Re\left[p_{n}(t)\right]
\end{aligned}\right.
$$




\section{II.B Linear stability analysis}

The model described above has a variety of possible behaviours. One of them is a static solution, all variables being constant. The stability of this static solution is a useful piece of information, as instability of the static solution indicates possible emergence of oscillating solutions through Hopf bifurcations. This stability analysis can be carried out on a linearised model: non-linear equations are linearised in the vicinity of the static solution. Then, the stability of this static solution is assessed through computation of the eigenvalues of the Jacobian matrix. If at least one eigenvalue has a positive real part, any perturbation of the static solution will grow exponentially, which by definition means the solution is unstable. Details on the method applied to brass instruments can be found in [Velut et al., 2017].

This method is used to find the lowest blowing pressure value leading to an unstable static solution. This $p_{b}$ value is hereafter called $p_{\text {thresh }}$. The imaginary part of the same eigenvalue indicates the oscillation angular frequency for $p_{b}=p_{\text {thresh }}$, provided that the oscillating solution is periodic. The corresponding frequency is noted $f_{\text {thresh }}$.

LSA has been used for flute-like instruments [Auvray et al., 2012, Terrien et al., 2014] as well as reed woodwinds [Wilson and Beavers, 1974, Chang, 1994, Silva et al., 2008] and brasswinds [Cullen et al., 2000, Velut et al., 2017]. This method does not provide information about the stability of the oscillating solution which results from the destabilisation of the static solution. The only piece of information about the resulting waveform is $f_{\text {thresh }}$, which is only valid if said solution is periodic.

An example of results is given in Fig. 3: $p_{\text {thresh }}$ (a) and $f_{\text {thresh }}$ (b) are plotted against the lip resonance frequency $f_{l}$, which is a control parameter used by the musician to change the note played with the trombone. As observed in [Velut et al., 2017], the plots can be divided in several $f_{l}$ ranges corresponding to U-shaped sections of the $p_{\text {thresh }}$ curves and very lightly growing plateaus of $f_{\text {thresh }}$ just above the acoustic resonance frequencies of the resonator. 

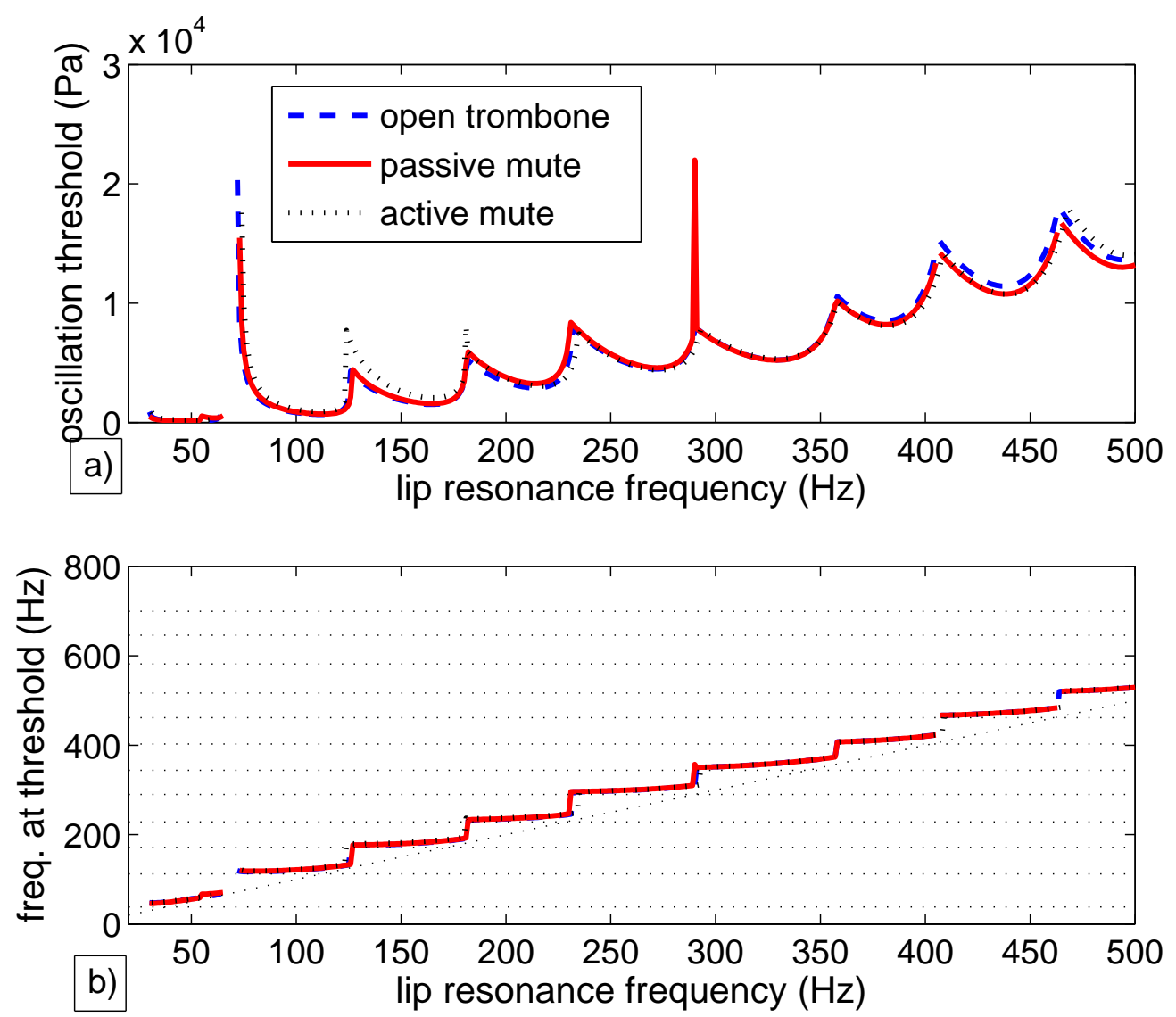

Figure 3: (colour online). Linear stability analysis results: $p_{\text {thresh }}$ (a) and $f_{\text {thresh }}$ (b) are plotted against $f_{l}$. Results for the open trombone (blue, dashed), the passive mute (red, solid) and the active mute (black, dotted) are displayed. Black dotted lines of the bottom plot are the resonance frequencies of the open trombone (horizontal) and the bisector of the axes $\left(f_{\text {thresh }}=f_{l}\right)$. The qualitative behaviour of the open trombone, the passive mute and active mute are very similar at this scale.

\section{II.C Time-domain simulation}

To get more information about the nature of oscillating solutions of the instrument model, solving the non-linear equation system Eq. (6) is required. Numerical differential equation solvers provide simulated values of the system variables. Simulated values of the pressure at the input of the instrument $p$ have been obtained with the open-source Python library called MoReeSC [Mor, 2016], which has been developed specially for time-domain simulation of self-oscillating reed and lip valve instrument models [Silva et al., 2014].

To illustrate the additional information provided by time-domain simulation, waveforms and spectra of two simulated pressure signals are given in Figure 4. The simulation in Fig. 4 (a) and (c) was computed with $f_{l}=90 \mathrm{~Hz}$ while the one in Fig. 4 (b) and (d) was computed with $f_{l}=110 \mathrm{~Hz}$, each one on an open trombone, with a blowing pressure $10 \%$ higher than the oscillation threshold. While LSA results for these two situations are very close to one another, numerical resolution of 
the complete model shows a difference in the nature of the oscillation: while the oscillation is periodic for $f_{l}=90 \mathrm{~Hz}$, it appears to be quasi-periodic for $f_{l}=110 \mathrm{~Hz}$.

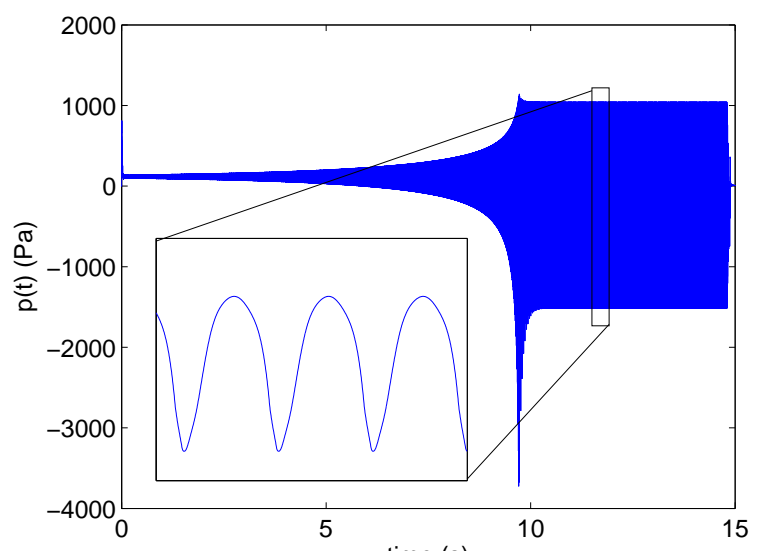

a)

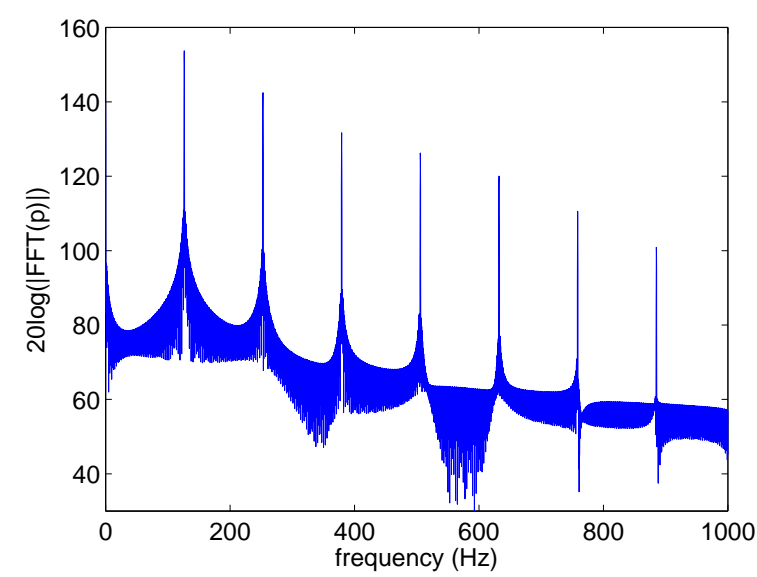

b)

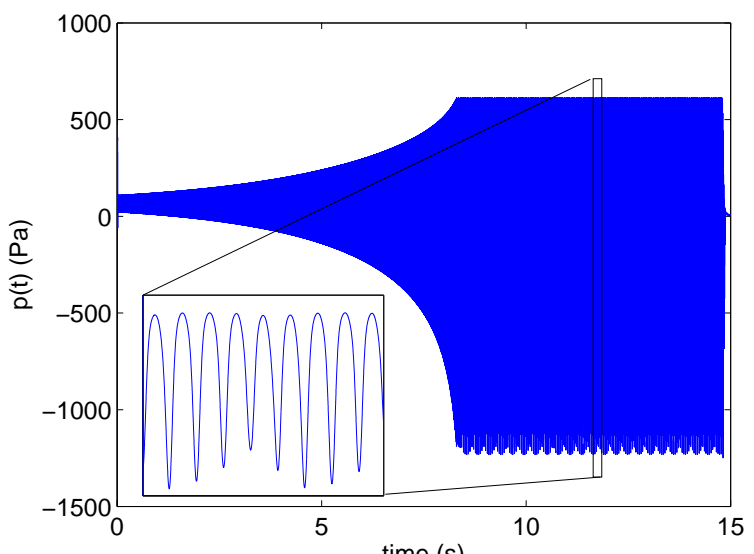

d)

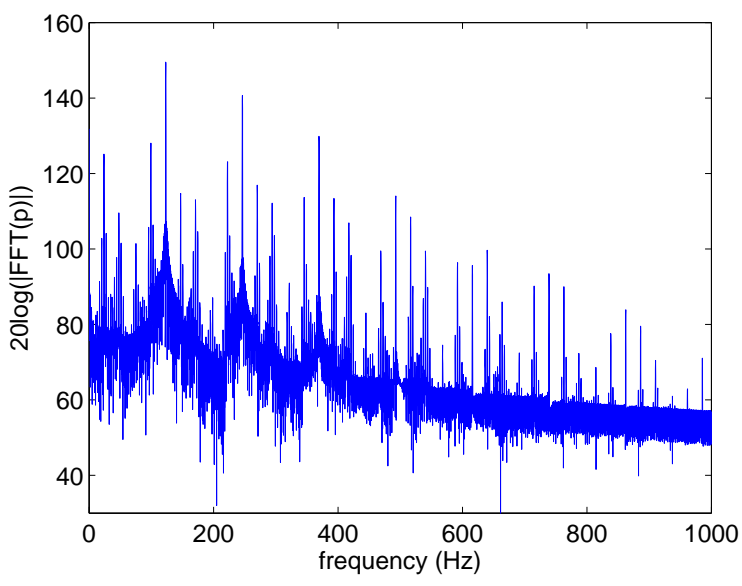

Figure 4: Waveforms of simulated $p$ signals for $f_{l}=90 \mathrm{~Hz}$ (a) and $f_{l}=110 \mathrm{~Hz}$ (b) with zooms on some periods, along with spectra of their respective sustained regime in c) and d). For each simulation $p_{b}$ is set to1.1 $p_{\text {thresh. }} . f_{l}=90 \mathrm{~Hz}$ results in a periodic oscillation while $f_{l}=110 \mathrm{~Hz}$ results in a quasi-periodic oscillation with well defined secondary peaks.

The $f_{l}$ and $p_{b}$ values for simulations are chosen thanks to LSA, avoiding a long and cumbersome search for the oscillation threshold with multiple simulations. The complementarity of these methods quickly provides a lot of information about relevant points of the oscillation regime.

\section{Results}

\section{III.A LSA}

Linear stability analysis was performed on the three configurations studied: open trombone, passive mute, and active mute. Choosing a configuration involves choosing $C_{n}$ and $s_{n}$ values among the three sets obtained by fitting, all other parameters of the model remaining the same. Lip parameters were taken from Table I. LSA was performed within the pedal note range, for $f_{l}$ from 
$30 \mathrm{~Hz}$ to $65 \mathrm{~Hz}$. This results in $f_{\text {thresh }}$ values corresponding to an oscillation sustained by the first acoustical mode of the open trombone. Figure 5 is a zoom on Fig 3 in the considered $f_{l}$ range. Fig. 5a) showing the threshold pressures $p_{\text {thresh }}$, while Fig. $5 \mathrm{~b}$ ) is the frequency at threshold $f_{\text {thresh }}$.
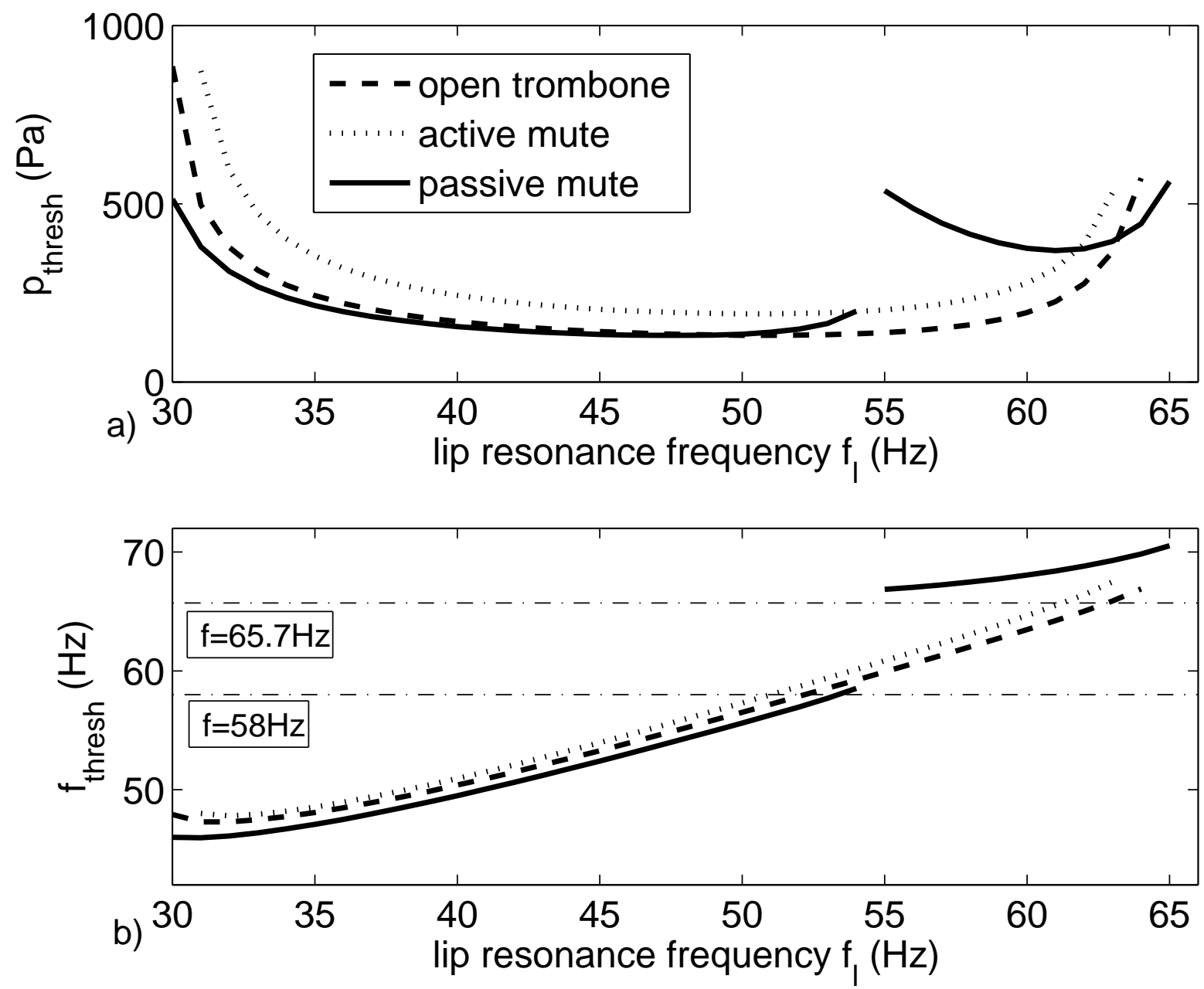

Figure 5: Results of LSA in the vicinity of the pedal note (zoom of Fig.3). Results with an open trombone (dashed line), a passive mute (solid line) and the active mute (dotted) are plotted together. (a) is the oscillation threshold pressure $p_{\text {thresh }},(\mathrm{b})$ is the oscillation frequency at threshold $f_{\text {thresh }}$, against $f_{l}$. Horizontal dash-dotted lines in (b) indicate $58 \mathrm{~Hz}$ (playing frequency of the pedal $B b$ ) and $65.7 \mathrm{~Hz}$ (resonance frequency of the subsidiary mode of the passive mute). While open trombone and active mute have very similar behaviours, the oscillation regime expected for the trombone with the passive mute becomes different above $f_{l}=55 \mathrm{~Hz}$ with a sudden increase in the $p_{\text {thresh }}$ and $f_{\text {thresh }}$ values.

The open trombone and the active mute behaviours are similar: the $p_{\text {thresh }}=F\left(f_{l}\right)$ plot is Ushaped. $f_{\text {thresh }}$ is above the trombone's first acoustical resonance frequency (39 Hz) and increases monotonically with $f_{l}$. Within this $f_{l}$ range, the oscillation threshold of the active mute trombone is about $75 \mathrm{~Pa}$ higher than that of the open trombone, and $f_{\text {thresh }}$ is also 0.5 to $1.5 \mathrm{~Hz}$ higher.

For $f_{l} \leq 54 \mathrm{~Hz}$, the results for the trombone with a passive mute are similar to those for the other 
configurations. But from $f_{l}=55 \mathrm{~Hz}$, both the pressure threshold and the expected playing frequency increase significantly: $p_{\text {thresh }}$ suddenly jumps from 198.6 to $536.9 \mathrm{~Pa}$, while $f_{\text {thresh }}$ increases by $8.3 \mathrm{~Hz}(13 \%$, i.e. slightly more than a tone), to reach $66.8 \mathrm{~Hz}$. This value is just above the resonance frequency of the subsidiary peak induced by the passive mute.

$f_{\text {thresh }}$ covers a range of frequencies around the expected playing frequency of a pedal $B b=58$ Hz. The results for the open trombone and the active mute configurations are very close to one another, the only difference being a rather small offset in $p_{\text {thresh }}$ and $f_{\text {thresh. }}$. In contrast, the passive mute results stand out from the two other configurations: for $f_{l}$ values above $55 \mathrm{~Hz}$, $p_{\text {thresh }}$ and $f_{\text {thresh }}$ increase suddenly. The $f_{\text {thresh }}$ value obtained is above the acoustic resonance frequency of the subsidiary mode related to the passive mute, and so the regeneration condition [Eliott and Bowsher, 1982, Campbell, 2004] is satisfied for an oscillation supported by this subsidiary mode.

These results can account for the difficulty of playing a stable pedal note with a passive mute: the LSA indicates a perturbation of the oscillation frequency at threshold, for parameters which could be those used for the pedal note. However, experimental results shown in [Meurisse et al., 2015] suggest a non-periodic oscillation when a musician tries to play a pedal note with a passive mute. As LSA cannot predict the nature of the oscillation, further investigation on the complete nonlinear model is needed. This is the purpose of the numerical simulations presented in the following section.

\section{III.B Time-domain simulations}

Time-domain simulations were carried out within the same range of $f_{l}$ as for LSA, in $1 \mathrm{~Hz}$ steps, for each configuration: trombone alone, trombone with a passive mute and finally trombone with active mute. The blowing pressure was set to $p_{b}=1.1 \cdot p_{\text {thresh }}$ as in [Velut et al., 2017] in order to keep manageable transient times. This value is close enough to $p_{\text {thresh }}$ so that cautious comparisons can be carried out between these simulations and LSA.

Simulated pressure signals were separated into a transient and a sustained regime with the help of the "mironsets" function from MIRtoolbox [Lartillot and Toiviainen, 2007]. The spectra of all the sustained regimes were computed. Figure 6 plots spectra of $\mathrm{p}(\mathrm{t})$ for representative values of $f_{l}$. 

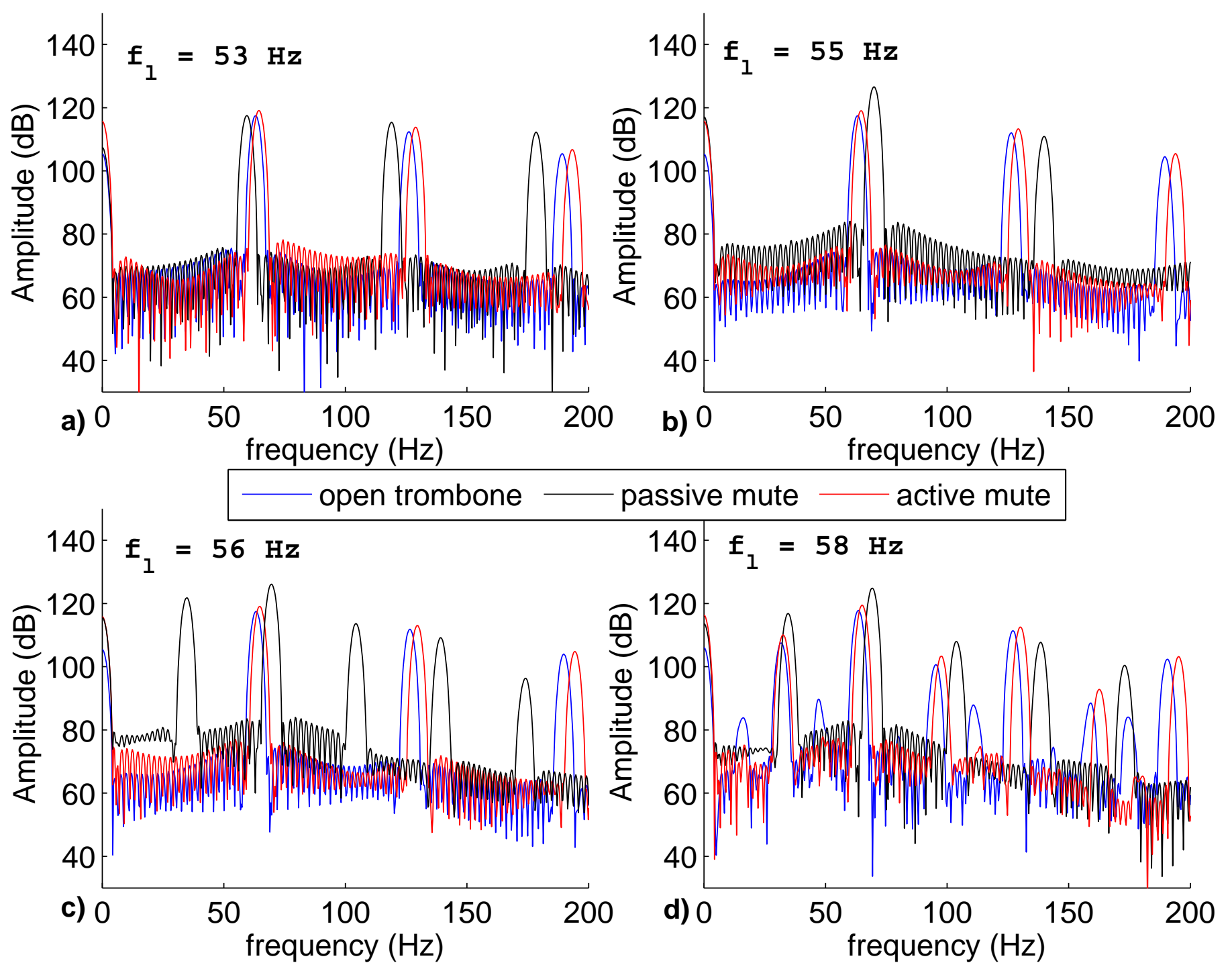

Figure 6: (Colours online) Spectra of the simulated $p(t)$ signals, for $f_{l}=53 \mathrm{~Hz}$ (a), $55 \mathrm{~Hz}$ (b), 56 $\mathrm{Hz}(\mathrm{c})$ and $58 \mathrm{~Hz}(\mathrm{~d}) . p_{b}$ is set to $110 \%$ of the oscillation threshold. For each $f_{l}$ value, results with the open trombone (blue), the passive mute (black) and the active mute (red) are displayed. The results for the open trombone and the active mute are noticeably similar.

For $f_{l}<55 \mathrm{~Hz}$, the three configurations - open trombone, passive mute, active mute - lead to a periodic oscillation, as illustrated for $f_{l}=53 \mathrm{~Hz}$ by (Fig. 6a). The oscillation frequency is a bit higher than $f_{\text {thresh }}$ : $7.5 \%$ for open trombone and active mute, and $2.5 \%$ higher for the passive mute. Oscillation frequencies higher than $f_{\text {thresh }}$ when $p_{b}>p_{\text {thresh }}$ is coherent with the fact that a musician's playing frequency gets higher when the blowing pressure increases. The trombone with a passive mute has a lower oscillation frequency than the open trombone, which has itself a slightly lower oscillation frequency than that of the trombone with the active mute. The oscillation frequencies range from 60 to $64 \mathrm{~Hz}$, a bit higher than $B b 1=58 \mathrm{~Hz}$. This is sensible since this model is known to oscillate at higher frequencies than those at which a musician plays.

At $f_{l}=55 \mathrm{~Hz}$ (Fig. 6b) the oscillation frequency of the passive mute configuration suddenly jumps from 59.4 to $69.6 \mathrm{~Hz}$, making it play sharper (nearly a minor third) than the two other 
configurations. This is consistent with the LSA results, where $f_{\text {thresh }}$ suddenly increases by $8 \mathrm{~Hz}$ for this $f_{l}$ value. The oscillations are still periodic and above the acoustical resonance frequency of the first mode; but for $f_{l}=56 \mathrm{~Hz}$ and above (illustrated by Fig. 6c) the fundamental frequency of the passive mute falls to $34.8 \mathrm{~Hz}$. This is nearly half its former value, and under the trombone's first acoustic resonance frequency $\left(39 \mathrm{~Hz}\right.$ ). Finally, for $f_{l} \geq 58 \mathrm{~Hz}$ (Fig. 6d), all configurations result in fundamental oscillation frequencies about half, or a quarter, of the oscillation frequencies obtained for lower $f_{l}$ values.

Simulation and LSA results are consistent: when $f_{l}$ reaches $55 \mathrm{~Hz}$, the oscillation frequency of the trombone with a passive mute suddenly increases. This is related to a regime change in the instrument: for $f_{l}<55 \mathrm{~Hz}$, the oscillation is mainly supported by the trombone's first acoustical mode which resonance frequency is $38 \mathrm{~Hz}$. The unusal gap between the trombone's first mode and the pedal note is studied in [Velut et al., 2017]. For $f_{l}=55 \mathrm{~Hz}$ and above, the subsidiary mode at $65.7 \mathrm{~Hz}$ caused by the mute becomes the main supporting mode of the oscillation, which explains the increase in the oscillating frequency.

Above $f_{l}=56 \mathrm{~Hz}$, however, the oscillation frequency of the passive mute decreases to half of its former value. As the oscillation frequency is under the trombone's first acoustical resonance frequency, the regeneration condition of a model with outward-striking valve is not satisfied [Campbell, 2004]. This situation suggests a period-doubling phenomenon [Bergé et al., 1995]. When increasing $f_{l}$ again, the three configurations appear to undergo period doubling, which is further doubled for the open trombone with a fundamental frequency of $16.2 \mathrm{~Hz}$. Sub-harmonic cascades have already been observed for trombones [Gibiat and Castellengo, 2000], and simulated in a previous study with the very same model and parameters [Velut et al., 2017].

These results confirm the existence of a subsidiary regime of oscillation for the passive mute configuration, which could explain why musicians experience difficulties when trying to play the $B b$ pedal in this situation. This subsidiary regime is sustained by the subsidiary acoustical mode introduced by the mute. Furthermore, in accordance with the experimental results published in [Meurisse et al., 2015], the simulation results are qualitatively the same for the open trombone and the active mute, with very close oscillation frequencies. The range of $f_{l}$ leading to periodic oscillations near the pedal note frequency is noticeably wider for the open trombone and the active mute than for the passive mute.

\section{Conclusion}

Playing a stable $B b 1$ on a trombone with a straight mute is very difficult. An active mute has been developed [Meurisse et al., 2015] to deal with this issue. When applied to a trombone equipped with this active mute and to an open trombone, LSA and time-domain simulation give nearly identical results. The model is therefore able to predict the efficiency of the active control device which makes the pedal note easily playable again. Results of the model of a trombone equipped with a passive mute, however, are clearly different from those of the open trombone model: the 
pedal note is disturbed by a new oscillation regime, which seems related to the subsidiary acoustical mode added by the mute. Hence, even a "small" perturbation of the input impedance, such as a peak 20 times smaller in amplitude than surrounding peaks, can strongly affect the behaviour of a resonator.

As in a previous paper [Velut et al., 2017], this study shows a rather good agreement between LSA results and time-domain simulations, within the limits of the LSA method. This study on mutes also shows the relevance of the chosen brass instrument model, which is able to predict a number of behaviours of the trombone, including particular playing regimes [Velut et al., 2017, Velut et al., 2016a] and, in the present case, the influence of modifications of the instrument bore. Beginning a study with LSA very quickly gives an overview of the potential behaviour of the system under given conditions. This fast computation already provides interesting results, which can be interpreted alone. However, if further exploration of the oscillation regime is required, LSA results give hints for choosing $f_{l}$ and $p_{b}$ values for initialising other analysis methods.

\section{Acknowledgements}

The authors would particularly like to thank Thibault Meurisse and Adrien Mamou-Mani for providing the input impedance measurements used here.

This work was done in the frameworks of the Labex MEC (ANR-10-LABX-0092) and of the $\mathrm{A}^{*}$ MIDEX project (ANR-11-IDEX-0001-02), funded by the French National Research Agency (ANR).

\section{References}

[Mor, 2016] (2016). Source website of the moreesc software. http://moreesc.lma.cnrs-mrs.fr/. Accessed: 2015-06-22.

[Adachi and Sato, 1996] Adachi, S. and Sato, M. (1996). Trumpet sound simulation using a twodimensional lip vibration model. J. Acoust. Soc. Am., 99(2):1200-1209.

[Auvray et al., 2012] Auvray, R., Fabre, B., and Lagrée, P. (2012). Regime change and oscillation thresholds in recorder-like instruments. J. Acoust. Soc. Am., 131(2):1574-1585.

[Backus, 1976] Backus, J. (1976). Input impedance curves for the brass instruments. J. Acoust. Soc. Am., 60(2):470-480.

[Bergé et al., 1995] Bergé, P., Pomeau, Y., and Vidal, C. (1995). L’odre dans le chaos: vers une approche déterministe de la turbulence. Hermann.

[Campbell, 2004] Campbell, M. (2004). Brass instruments as we know them today. Acta Acustica united with Acustica, 90:600-610. 
[Campbell and Greated, 1994] Campbell, M. and Greated, C. (1994). The musician's guide to Acoustics. Oxford Universitary Press, Oxford.

[Chang, 1994] Chang, Y. M. (1994). Reed stability. Journal of Fluids and Structures, 8:771-783.

[Cullen et al., 2000] Cullen, J., Gilbert, J., and Campbell, M. (2000). Brass instruments: Linear stability analysis and experiments with an artificial mouth. Acta Acustica united with Acustica, $86: 704-724$.

[Eliott and Bowsher, 1982] Eliott, S. and Bowsher, J. (1982). Regeneration in brass instruments. Journal of Sound and Vibration, 83:181-217.

[Fletcher, 1993] Fletcher, N. (1993). Autonomous vibration of simple pressure-controlled valves in gas flows. J. Acoust. Soc. Am., 93(4):2172-2180.

[Gibiat and Castellengo, 2000] Gibiat, V. and Castellengo, M. (2000). Period doubling occurences in wind instruments musical performance. Acta Acustica united with Acustica, 86:746-756.

[Gilbert and Aumond, 2008] Gilbert, J. and Aumond, P. (2008). Pedal notes of brass instruments, a mysterious regime of oscillation. In Proceedings of Acoustics' 08, Paris.

[Lartillot and Toiviainen, 2007] Lartillot, O. and Toiviainen, P. (2007). A matlab toolbox for musical feature extraction from audio. In Proc. of $10^{\text {th }}$ International Conference on Digital Audio Effects (DaFx), Bordeaux, France.

[Lopez et al., 2006] Lopez, I., Hirschberg, A., Hirtum, A. V., Ruty, N., and Pelorson, X. (2006). Physical modeling of buzing artificial lips: The effect of acoustical feedback. Acta Acustica united with Acustica, 92:1047-1059.

[Macaluso and Dalmont, 2011] Macaluso, C. and Dalmont, J.-P. (2011). Trumpet with nearperfect harmonicity: Design and acoustic results. J. Acoust. Soc. Am, 129(1):404-414.

[Meurisse et al., 2015] Meurisse, T., Mamou-Mani, A., Caussé, R., Sluchin, B., and Sharp, D. (2015). An active mute for the trombone. J. Acoust. Soc. Am., 138(6):3539-3548.

[Myers et al., 2012] Myers, A., Pyle, R., Gilbert, J., Campbell, M., Chick, J., and Logie, S. (2012). Effects of nonlinear sound propagation on the characteristic timbres of brass instruments. $J$. Acoust. Soc. Am., 131(1):678-688.

[Newton et al., 2008] Newton, M., Campbell, M., and Gilbert, J. (2008). Mechanical response measurements of real and artificial brass players lips. J. Acoust. Soc. Am., 123(1):EL14-EL20.

[Silva, 2009] Silva, F. (2009). Émergence des auto-oscillations dans un instrument de musique à anche simple. PhD thesis, Universite de Provence Aix-Marseille 1. 
[Silva et al., 2007] Silva, F., Kergomard, J., and Vergez, C. (2007). Oscillation thresholds for "striking outwards" reeds coupled to a resonator. In Proceedings of ISMA 200\%, Barcelona.

[Silva et al., 2008] Silva, F., Kergomard, J., Vergez, C., and Gilbert, J. (2008). Interaction of reed and acoustic resonator in clarinet-like systems. J. Acoust. Soc. Am., 124(5):3284-3295.

[Silva et al., 2014] Silva, F., Vergez, C., Guillemain, P., Kergomard, J., and Debut, V. (2014). MoReeSC : a framework for the simulation and analysis of sound production in reed and brass instruments. Acta Acustica United with Acustica, 100(1):126-138.

[Sluchin and Caussé, 1991] Sluchin, B. and Caussé, R. (1991). Sourdines des cuivres (Brass instrument mutes). Editions de la Maison des Sciences de l'Homme, Paris.

[Terrien et al., 2014] Terrien, S., Vergez, C., and Fabre, B. (2014). To what extent can a linear analysis predict the behaviour of a flute model? In Proceedings of International Symposium on Musical Acoustics, Le Mans, France.

[Velut et al., 2016a] Velut, L., Vergez, C., and Gilbert, J. (2016a). Measurements and time-domain simulations of multiphonics in the trombone. hal-01363547; Submitted to JASA, currently in revision: https://hal.archives-ouvertes.fr/hal-01363547.

[Velut et al., 2017] Velut, L., Vergez, C., Gilbert, J., and Djahanbani, M. (2017). How well can linear stability analysis predict the behavior of an outward valve brass instrument model? Acta Acustica united with Acustica, 103(1):132-148. https://doi .org/10.3813/AAA.919039.

[Velut et al., 2016b] Velut, L., Vergez, C., Gilbert, J., Mamou-Mani, A., and Meurisse, T. (2016b). Effets d'une sourdine sur le seuil et la fréquence d'oscillation du premier régime du trombone (effects of a mute on the threshold and the oscillation frequency of the trombone's first register). In Proceedings of CFA/VISHNO 2016, page 313, Le Mans, France. http://www.conforg.fr/ cfa2016/cdrom/data/articles/000057.pdf.

[von Helmholtz, 1870] von Helmholtz, H. (1870). On the Sensations of Tone. Dover Publications Inc., New York.

[Wilson and Beavers, 1974] Wilson, T. and Beavers, G. (1974). Operating modes of the clarinet. J. Acoust. Soc. Am., 56(2):653-658.

[Yoshikawa, 1995] Yoshikawa, S. (1995). Acoustical behavior of brass player's lips. J. Acoust. Soc. Am, 97(3):1929-1939. 